\title{
Intrinsic dorsoventral patterning and extrinsic EGFR signaling genes control glial cell development in the
} Drosophila nervous system

Hyeon Ju Kim', Hui Jeong Ahn', Sihaeng Lee ${ }^{1}$, Jung Ho Kim¹, Jiseung Park ${ }^{1}$, Sang-Hak Jeon $^{2}$ and Sang Hee $\mathrm{Kim}^{1} *$

${ }^{1}$ Department of Chemistry, Konkuk University, Seoul 143-701, Korea,

${ }^{2}$ Department of Biology Education, Seoul National University, Seoul 151-742, Korea

*Author for correspondence

Tel: 82-2-450-3439

Fax: 82-2-444-8344

E-mail: flier@konkuk.ac.kr

Mailing address: Department of Chemistry

Konkuk University

120 Neungdong-ro Gwangjin-gu

Seoul 143-701, Korea 


\begin{abstract}
Dorsoventral patterning and EGFR signaling genes are essential for determining neural identity and differentiation of the Drosophila nervous system. Their role in glial cell development in the Drosophila nervous system is not clearly established. Our study demonstrated that the dorsoventral patterning genes, $v n d$, ind, and $m s h$, are intrinsically essential for the proper expression of a master glial cell regulator, $\mathrm{gcm}$, and a differentiation gene, repo, in the lateral glia. In addition, we showed that esg is particularly required for their expression in the peripheral glia. These results indicate that the dorsoventral patterning and EGFR signaling genes are essential for identity determination and differentiation of the lateral glia by regulating proper expression of $\mathrm{gcm}$ and repo in the lateral glia from the early glial development. In contrast, overexpression of $v n d, m s h$, spi, and Egfr genes repressed the expression of Repo in the ventral neuroectoderm, indicating that maintenance of correct columnar identity along the dorsoventral axis by proper expression of these genes is essential for restrictive formation of glial precursor cells in the lateral neuroectoderm. Therefore, the dorsoventral patterning and EGFR signaling genes play essential roles in correct identity determination and differentiation of lateral glia in the Drosophila nervous system.
\end{abstract}

\footnotetext{
Key words: Drosophila, glia, development, dorsovental patterning, EGFR signaling genes
} 


\section{INTRODUCTION}

The nervous system is composed of two types of cells: neurons and glia. Glia are known to constitute more than $50 \%$ of the mammalian brain and play diverse roles in the development and function of the nervous system (Villegas et al., 2003; Rowitch and Kriegstein, 2010; Verkhratsky et al., 2010; Stork et al., 2012). They provide structural support, insulate neurons, and regulate their differentiation and survival through cytokines and growth factors. They also form the blood-brain barrier and regulate the extracellular concentrations of ions and neurotransmitters to make the environment suitable for propagation of electrical signals. In addition, they guide developing neurons and their axons to their targets, and play important roles in modulating synaptic activity and regulating synapse numbers (Barres, 2008; Halassa and Haydon, 2010). Finally, they mediate inflammation in the central nervous system (CNS), and clear dead cell debris (Lampron et al., 2013; Salter and Beggs, 2014).

Mammalian gliogenesis is regulated by an intricate interplay between intrinsic transcription factors and extrinsic signaling pathways (Miller and Gauthier, 2007; Rowitch and Kriegstein, 2010). Homeodomain-containing patterning genes, such as Otx, Gbx, and Hox along the anteroposterior (AP) axis in the brain and Nkx, Dbx, Irx, and Pax along the dorsoventral (DV) axis in the spinal cord, intrinsically establish regional identity, such as pMN that generates both motoneurons and oligodendrocytes. At the same time, extrinsic signaling molecules, such as bone morphogenetic protein (BMP), cytokines, receptor tyrosine kinase (RTK), Shh, and Wnt, work together to activate spatially and temporally lineagespecific genes that establish regulatory networks for maintaining a particular identity. Combination of different transcription factors within the parental cell results in cell-specific codes and different arrays of genes in the astrocytes and oligodendrocytes. These hierarchical regulatory interactions occur within spatial and temporal contexts of the signaling molecules 
to generate unique cell identities.

The fruit fly Drosophila has provided a wealth of information on the molecular mechanisms of gliogenesis and their cellular function relevant to vertebrates (reviewed by Jones, 2001; Chortard and Salecker, 2004; Edenfeld et al., 2005; Freeman and Doherty, 2006; Parker and Auld, 2006; Hartenstein, 2011; Stork et al., 2012). Much information was obtained for identities and lineages of neurons and glia and for the pathways taken by axons in the developing CNS and peripheral nervous system (PNS) (Ito et al., 1995; Bossing et al., 1996; Schmidt et al., 1997; Schmid et al., 1999; Beckervordersandforth et al., 2008; von Hilchen et al., 2008). The embryonic CNS develops from stem cells of the ventral neuroectoderm (VNE) by delamination in five fixed waves and at defined positions. Stem cells consist of 21 neuroblasts (NBs), 7-8 neuroglioblasts (NGBs), and a glioblast (GB) per hemisegment (Hartenstein, 2011; Stork et al., 2012). NBs produce only neurons, GBs only glia, and NGBs both neurons and glia (Soustelle and Giangrande, 2007). NBs produce ganglion mother cells (GMCs) each time by 1-12 successive asymmetric cell divisions and finally divide to yield about 350 neurons and 30 glia per hemisegment. Most glial cells are derived from the lateral neuroectoderm located on either side of the CNS midline and are therefore, called lateral glia (LTG). Another class, the midline glia, is derived from the mesectoderm, which has a structure similar to the floor plate in the vertebrate spinal cord. Their identity and function differ from the LTG. The LTG are composed of the neuropile glia (NG), including the longitudinal glia (LG) that are associated with the longitudinal connectives, the peripheral glia (PG) that migrate out and ensheath the peripheral neurons, and the exit glia (EG) that are located at the junction of the segmental and intersegmental nerves, the surface glia (SG), including A and B glia, which are located at the anterior and posterior commissures, and the cortex glia (CG).

Drosophila NB identity relies on precise positional and temporal information along the 
AP and DV axes (reviewed by Bhat, 1999; Skeath, 1999; Kohwi and Doe, 2013). Extrinsic $\mathrm{BMP} / \mathrm{Dpp}, \mathrm{NF}-\kappa \mathrm{B} /$ Dorsal, and epidermal growth factor receptor (EGFR) signaling act together to subdivide the VNE into three columns along the DV axis. They achieve this by regulating the expression of three DV patterning homeodomain genes, ventral nervous system defective (vnd) (Jimenez et al., 1995; Mellerick and Nirenberg, 1995; Chu et al., 1998; McDonald et al., 1998), intermediate neuroblasts defective (ind) (Weiss et al., 1998), and muscle-specific homeodomain (msh) (D’Alessio and Frasch, 1996; Wang et al., 1996; Isshiki et al., 1997; reviewed by Cornell and von Ohlen, 2000; Skeath and Thor, 2003). In addition, one of the snail family genes, escargot (esg), is known to encode related zinc finger transcription factors that mediate Drosophila CNS development through asymmetric division of NBs (Ashraf et al, 1999; Ashraf and Ip, 2001; Cai et al., 2001). The master gene for development of the LTG is the glial cells missing/glial cell deficient $(\mathrm{gcm})$. It encodes a novel transcription factor with Gcm-binding motifs that is transiently expressed in all glia except for the midline glia. $\mathrm{gcm}$ functions as a binary genetic switch for glia versus neurons: glia are transformed into neurons in the $\mathrm{gcm}$ mutant embryos, whereas presumptive neurons become glia when $\mathrm{gcm}$ is ectopically expressed (Hosoya et al., 1995; Jones et al., 1995; Vincent et al., 1996; Mao et al., 2012). Although it is well known how $\mathrm{gcm}$ determines glial identity and control glial differentiation by transcriptional activation of downstream target genes, relatively little is known about how spatial and temporal identity determination of glial cells is intrinsically and extrinsically regulated by the DV patterning and EGFR signaling genes.

In this study, several glial cell markers were employed to investigate how the DV patterning and EGFR signaling genes are intrinsically and extrinsically involved in identity determination and in differentiation of the LTG. This study revealed that the DV patterning genes are required for proper identity determination and differentiation of the LTG in the corresponding columns of the VNE. EGFR signaling genes also play important roles in the 
identity determination and differentiation of the LTG in the entire VNE. We conclude that the DV patterning and EGFR signaling genes intrinsically and extrinsically control correct identity determination and differentiation of the LTG.

\section{EXPERIMENTAL PROCEDURES}

\section{Fly strains}

The following DV patterning and EGFR signaling mutants were used as loss-of-function mutants: Egfr ${ }^{f 7}$, esg ${ }^{G 66}$, ind $^{R R 108}, \mathrm{msh}^{468}$, single-minded $(\operatorname{sim})^{2}$, spitz $(\text { spi })^{1}$, and $v n d^{6}$. All genetic markers and balancer chromosomes used are as described in FlyBase (www.flybase.com). For overexpression of the DV patterning and Egfr signaling genes, Krüppel (Kr)-Gal4 (Brand and Perrimon, 1993), scabrous (sca)-Gal4 (Klaes et al., 1994), $U A S$-msh (Isshiki et al., 1997), UAS-vnd (Chu et al., 1998), UAS-spi (Golembo et al., 1996), and UAS-vein (vn) (Schnepp et al., 1996) were used.

\section{Immunohistochemistry and whole mount in situ hybridization}

Antibody staining was carried out as described by Patel (1994). The following antibodies were obtained from the Developmental Studies Hybridoma Bank (DSHB) and used to detect axonal tracts and glia: monoclonal antibodies BP102 (1:20), 22C10 (1:30), and anti-Reverse polarity (Repo, 1:5). Mouse anti-Engrailed (En) antibody (DSHB) was used at a 1:20 dilution to mark segmental borders. Anti-phospho-MAPK antibody (Invitrogen) was used at 1:300 dilution. $g \mathrm{~cm}$ cDNA was used for analyzing the formation of glial cells. vnd cDNA (McDonald et al., 1998) was used as a fluorescent in situ hybridization probe. Whole-mount in situ hybridization using digoxigenin-labeled RNA probes was performed as described by O'Neill and Bier (1994). Antibody staining and the consecutive in situ hybridization, and 
regular antibody staining were carried out as described by Lehman and Tautz (1994) and Patel (1994), respectively. Fluorescence detection of digoxigenin-labeled RNA probes was carried out according to the manufacturer's instructions using the TSA amplification kit with Alexa 488 (Invitrogen). Stained embryos were viewed using an Olympus BX51 microscope with Nomarski optics, and FX-100 microscope, photographed with a Leica DC480 digital camera, and the images were processed using Adobe Photoshop.

\section{Quantitative analysis of the glial marker expression}

The number of gcm-positive and Repo-positive glial cells in the wild type, various DV patterning gene mutants, and UAS/Gal4 embryos was manually counted in each hemisegment of 3-5 embryos. The average number and its standard deviation were obtained by using Window Excel program.

\section{RESULTS}

\section{DV patterning genes are required locally for identity determination and differentiation} of the LTG

To investigate how the DV patterning and EGFR signaling genes influence identity determination and formation of glial cells, expression of the master glial cell identity marker, gcm, was examined in vnd, ind, msh, and esg mutants. In the wild type embryos, $\mathrm{gcm}$ expression began in the peripheral glioblasts (PGBs) at stage 8 , and was then followed by expression in the LGBs and PGBs at stage 10 (Fig. 1A, F). At stage 13, gcm was expressed in divided LG along the longitudinal tracts and in the laterally stretched NG (Fig. 1K). At stage 10, the number of $\mathrm{gcm}$-expressing PGBs in the wild type embryos started to fall from 12.0 to $10.7 \pm 3.1,11.0 \pm 1.0,9.7 \pm 2.1$, and $8.0 \pm 5.3$ in each segment of $v n d$, ind, $m s h$, and esg 
mutant embryos, respectively (the number of hemisegments, $n=36$; Fig. 1G-J). In addition, the number of $\mathrm{gcm}$-expressing LGBs fell from 12.0 to $6.3 \pm 1.2,12.0,4.3 \pm 1.2$, and $6.3 \pm 3.2$, respectively, in each segment of the mutant embryos ( $\mathrm{n}=36$; Fig. 1G-J). At stage 13, $\mathrm{gcm}$ expression in most of the bilaterally symmetric LG was absent, the number of NG was reduced, and the NG were closer to the midline in $v n d$ and $m s h$ embryos (Fig. $1 \mathrm{~L}, \mathrm{~N}$ ). In contrast, the LG and NG were almost similarly generated in ind and esg embryos as in the wild type (Fig. 1M, O).

To investigate the precise role of each DV patterning gene in glial cell differentiation, Repo expression was examined. In wild type embryos, Repo expression began in the LGBs of each segment at stage 11, and in divided LG and in laterally stretched NG at stage 12 (Fig. 2A, F). Repo was expressed in the LG along the longitudinal tracts, in the SG of the entire ventral nerve cord (VNC), and in the PG stretched out dorsally, as shown in each segment at stage 14 (Fig. 2K). The number of Repo-expressing LGBs was reduced to $14.2 \pm 4.0,17.6 \pm$ 1.8, and $19.4 \pm 1.7$ in vnd, $m s h$, and esg mutant embryos, from 28.0 in the wild type embryos $(n=60)$, respectively at stage 11 (Fig. $2 B, D, E)$, as was shown by examination of gcm expression. At stage 12, the number of Repo-expressing LG and NG was much reduced in the vnd embryos (Fig. 2G). Later at stage 14, most of the Repo-expressing LG were absent along the longitudinal tracts, and the PG were missing in some segments (Fig. 2L). In ind embryos, the number of Repo-expressing LG was not much changed as compared to that of the wild type embryos at stage 12 , but the numbers of NG and SG were reduced in the VNC, even though the LG and PG were regularly arranged along the longitudinal tracts and segmental nerve (Fig. 2M). In $m s h$ embryos, the LG did not properly form in the longitudinal tracts, and extra PG were present in the lateral neuroectoderm at stage 12 (Fig. 2I), but only the number of LG remained reduced (Fig. $2 \mathrm{~N}$ ) at stage 14. In stage 12 esg embryos, the numbers of LG and NG were slightly reduced at stage 14. In addition, the number of the PG 
was markedly reduced in the lateral region (Fig. 2O).

Taken together, these results indicated that $m s h$ and esg acted locally in the initial formation and differentiation of the LG from the lateral column of the VNC, and esg strongly influenced formation and differentiation of the PG. ind was also locally involved in the initial formation and differentiation of the NG and SG from the VNC. Unexpectedly, vnd, which is essential for identity determination of the medial column NBs, showed the strongest influence in the proper formation and differentiation of all glial cells including the LG, NG, SG, and even PG.

\section{EGFR signaling genes are broadly required for identity determination and differentiation of the LTG}

The influence of EGFR signaling genes on glial cell development was also investigated by examining the expression of general glial markers such as $g \mathrm{~cm}$ and Repo in sim, Egfr, and spi mutants. The number of $g \mathrm{~cm}$-expressing LGBs and PGBs was severely reduced in Egfr and spi mutant embryos at stage 10 (Fig. 3A, C, D). The number of Repo-expressing LGBs diminished from 28.0 in the wild type embryos to $15.6 \pm 2.5$ in the Egfr mutant embryos (n = $60)$ and $23.4 \pm 1.5$ in the spi mutant embryos $(\mathrm{n}=60)$ at stage 11 , respectively (Fig. 3E, G, H). Later, at stage 12, the number of Repo-expressing glial cells was dramatically reduced and was completely missing in some segments at stage 16 in the Egfr embryos (Fig. 3K, O). The spi embryos showed weaker defects in Repo expression than the Egfr mutants such that the overall expression pattern was maintained, although the number of Repo-expressing NG and SG in each segment was reduced at stage 12 and was almost absent in the VNC at stage 16 (Fig. 3L, P).

In contrast, expression of $\mathrm{gcm}$ and Repo in the LGBs and PGBs was not affected at stages 10 and 11, respectively, in sim embryos (Fig. 3B, F). The number of Repo-expressing LG and 
NG began to decreased at stage 12 (Fig. 3J), the LG were collapsed at the midline, and the numbers of LG and SG were significantly reduced at stage 16 (Fig. 3N). This result indicated that Egfr and spi are involved in both initial formation and differentiation of the LTG, but sim is only involved in differentiation.

\section{Overexpression of the DV patterning and EGFR signaling genes represses formation of the Repo-expressing LTG}

To investigate the effect of overexpression of the DV patterning genes on the formation of the LTG by examining expression of both Repo and activated MAPK, vnd was ectopically overexpressed in the VNE by crossing $K r$-Gal4 with $U A S$-vnd. When the medial column identity determinant, $v n d$, was ectopically overexpressed in the central region of the VNE in $K r$-Gal4/UAS-vnd embryos, the numbers of Repo-expressing LTG in the central region were reduced from $326 \pm 16$ in the wild type embryos into $142 \pm 32(n=5$ embryos $)$ at stage 15 (Fig. 4A, C, D, E, G, H). In addition, expression of activated MAPK in the longitudinal tracts, midline cells, segmental nerves and junctions between muscle and dorsal epidermis in wild type embryos was markedly reduced in Kr-Gal4/UAS-vnd embryos (Fig. 4B, F). Overexpression of msh by sca-Gal4 and $\mathrm{Kr}$-Gal4 drivers also showed similar result with lesser severity (data not shown). Overexpression of EGFR signaling ligands, Spi, in $\mathrm{Kr}$ Gal4/UAS-spi embryos also reduced the number of especially Repo-expressing NG and SG to $132 \pm 26$ (Fig. 4I-K). Similarly, overexpression of another EGFR signaling ligand, Vn, in the central region of the VNE by sca-Gal4 driver resulted in more severe reduction of the Repopositive NG and SG (data not shown).

These results suggest that overexpression of $v$ nd exerts the most severe reduction of the number of all Repo-expressing glia in the entire VNE, presumably due to identity change of NBs in the intermediate and lateral columns, where most of the LG, SG, and PG originate. 
On the other hand, overexpression of $m s h$ in the entire VNE affected Repo expression mainly in the SG. Overexpression of the EGFR signaling ligands, Spi and Vn, in the VNE also repressed Repo expression, particularly in the NG and SG, by expansion of the medial and intermediate identity into the lateral column region.

\section{Reduced expression of the glial cell marker, Repo, in the LTG is due to the defects in DV identity determination}

The experimental results obtained thus far strongly suggested that proper establishment and maintenance of the DV identity of the VNE are essential for glial development. To demonstrate that the overexpression of each DV patterning and EGFR signaling gene indeed represses Repo expression by identity change and thereby, inhibits glia formation and differentiation in the VNE, expressions of both Repo and vnd were examined after overexpression of $v n d$, driven by $\mathrm{Kr}$-Gal4. In stage 11 wild type embryos, $v n d$ was expressed in the medial neurons that are bilaterally located on each side of the VNE (Fig. 5A, C, G, I). On the other hand, Repo was expressed in the LGBs (Fig. 5B, C, H, I). In stage $11 \mathrm{Kr}$ Gal4/UAS-vnd embryos, vnd was expressed in the mesoderm of the embryos as well as in the medial neurons (Fig. 5D, F, J, L). In contrast, Repo expression was abolished in the $K r$ expressing central region of embryos (Fig. 5E, F, K, L). This result indicatedthat overexpression of $v n d$ in the $K r$-expressing region of the VNE transforms the identity of the lateral column, where LGBs originate, into medial column identity and thereby, Repo expression in the LGBs of each segment is abolished in that region.

To demonstrate further that the identity change in the VNE, caused by overexpression of $m s h$, vnd, Egfr, and spi, represses Repo expression, expressions of both Repo and activated MAPK were examined after overexpression of Egfr, $m s h$, spi, and $v n d$ in the VNE by driving by $K r$-Gal4. At stage 15, Repo was expressed in the LG that were located along the 
longitudinal tracts, in the SG of the entire VNC, and in the PG that were stretched out dorsally (Fig. 6A, B, D), and MAPK was expressed in each segmental boundary (Fig. 6A, C, D). In $K r$-Gal4/UAS-Egfr ${ }^{A C}$ and $K r$-Gal4/UAS-spi embryos, expressions of Repo and activated MAPK in the LTG were severely reduced in the $K r$-expressing middle region of the embryos (Fig. 6E-L). In $K r$-Gal4/UAS-vnd and $K r$-Gal4/UAS-msh embryos, expressions of Repo and activated MAPK were also reduced in the $K r$-expressing central region of embryos (Fig. 6M-T). These results suggested that the identity change within the VNE, monitored by activated MAPK expression, represses Repo expression in all the glial cells of the VNC, and that the proper expression of the DV patterning and EGFR signaling genes in the VNE is critical for the correct formation and differentiation of glial cells in the Drosophila nervous system.

\section{DISCUSSION}

Although it has been widely reported that DV patterning and EGFR signaling genes play essential roles in the proper identity determination and differentiation of NBs in the VNE, their roles in glial cell development have not been thoroughly studied to date. We clearly demonstrated that both DV patterning and EGFR signaling genes play essential roles in regulating the glial cell identity gene, $\mathrm{gcm}$, and the differentiation gene, repo, during Drosophila CNS development. Similar to their roles in neuronal development, DV patterning genes act locally in the regions where they are expressed, except for $v n d$, which influences formation and differentiation of glial cells in the entire VNE. EGFR signaling genes, however, influence glial cell formation and differentiation throughout the entire VNE, presumably due to long-range signal activation of EGFR signaling by diffusible ligands such as Spi and Vn. These results indicated that the DV patterning genes control gliogenesis locally in the corresponding columns, while the EGFR signaling genes broadly control gliogenesis 
throughout the entire VNE.

\section{Most DV patterning genes, except for $v n d$, are required locally for the proper formation and differentiation of glial cells in the corresponding VNE columns}

We demonstrated that the DV patterning genes, ind, $m s h$, and esg, are required for expression of the glial cell identity marker, $\mathrm{gcm}$, and of the glial cell differentiation marker, Repo, in the proper region of the LTG in the Drosophila VNE. msh and esg acted locally in the formation and differentiation of the LG from the lateral column of the VNE, and esg strongly influenced the formation and differentiation of the PG. ind was also locally involved in the initial formation and differentiation of the SG from the VNE (Figs. 1 and 2). Considering that DV patterning genes, such as ind and $m s h$, are required for the identity determination and formation of NBs in the intermediate and lateral columns along the DV axis, it is plausible that these two genes play essential roles in the proper development of the LTG in the corresponding columns. Interestingly, the zinc finger transcription factor, Esg, plays an important role in the formation and differentiation of the PG that originate from the lateral column, where esg is expressed (Fig. 1J, O and Fig. 2J, O). Although esg, together with snail and worniu, is required for the asymmetric division of $\mathrm{NBs}$, the precise role of esg in embryonic CNS development has not been clearly determined (Fuse et al, 1996; Ashraf et al., 1999; Ashraf and Ip, 2001; Cai et al, 2001). Thus, experimental results obtained in this study on esg's role in glial cell formation and differentiation is the first of its kind to analyze the role of esg in gliogenesis during embryonic CNS development.

Unexpectedly, vnd, which is essential for identity determination of the medial column NBs, showed the strongest influence on the proper formation and differentiation of all glia, including the LG, SG, and even PG in the VNE (Fig. 1G, L and Fig. 2B, G, L). Since the region of $m s h$ expression is ventrally expanded in the vnd mutant (Chu et al., 1998), 
disruption of the expression of $\mathrm{gcm}$ and Repo in the lateral column may have caused a decrease in the number of LG, LTG, and PG that originate from this region. In addition, the overexpression of $v n d$ also repressed the expressions of Repo and MAPK in the $K r$ domain, presumably by promoting identity determination of the medial column in the intermediate and lateral columns as shown in Figs. 5 and 6. Original reports on the role of the vnd in formation and identity determination of the medial column NBs using the vnd target gene, NK6, showed that intermediate and lateral column identity markers were repressed by overexpression of $v n d$ in the $\mathrm{Kr}$-expression domain (Chu et al., 1998; McDonalds et al., 1998; Uhler et al., 2002). One of the reasons for the wider influence of $v$ nd in DV patterning than other DV patterning genes may be that vnd is expressed earliest amongst these genes, repressing expression of other DV patterning genes such as ind and $m s h$ in the medial column, in a process termed "ventral dominance" (Cowden and Levine, 2003).

\section{Egfr signaling genes are broadly required for the proper formation and differentiation of glial cells throughout the VNE}

Our data revealed that the EGFR signaling receptor and ligand, Egfr and spi, play more global roles in glial cell development than do the DV patterning genes (Fig. 3). Egfr and spi are required for initial glial cell formation as shown by reduced expression of $\mathrm{gcm}$ and Repo in the LGBs of the VNE (Fig. 3F, G). In addition, Repo expression in the differentiated glia was markedly reduced, especially in Egfr embryos, and in spi embryos, to a lesser degree (Fig. 3O, P). Interestingly, Repo expression is almost absent in the SG and remains only in the LGs of spi as well as of ind embryos (Figs. 2M, 3P). Since ind expression is activated by the EGFR signaling ligand, Spi, in the VNE to establish the identity of the intermediate column, it is plausible that glial phenotypes in spi and ind mutants are similar to each other. This result indicated that once the intermediate column identity is determined by ind- 
mediated repression of $m s h$ expression in the lateral column, EGFR signaling provides a consolidating extrinsic cue to make ind a repressor of some of the target genes in the intermediate column via MAPK-mediated phosphorylation (Moses et al., 2011). This interpretation is compatible with the results obtained by overexpression of Spi and Vn through $\mathrm{Kr}$ - and sca-Gal4 drivers, which show repressed Repo expression in the VNE due to the repressor activity of Ind, which in turn is activated by EGFR signaling (Fig. 4I, K). Thus, our results indicated that EGFR signaling globally activates many types of glial cell lineages in the VNE and delimits the area where glial cells originate by repressor activity that is chemically modified by EGFR signal transduction.

\section{Establishment of proper identity along the DV axis by expression of the DV patterning and EGFR signaling genes is essential for correct formation and differentiation of glia from the VNE}

Our study revealed that the DV patterning and EGFR signaling genes play important roles in the initial formation and differentiation of various types of glia in the Drosophila CNS. Our data showed that the DV patterning genes and EGFR signaling genes are locally and globally required, respectively, for glial cell formation and differentiation using loss-of function mutants of the genes. Unexpectedly, overexpression of the DV patterning and EGFR signaling genes also repressed the initial formation and differentiation of glia, as shown in Figs. 4-6. Overexpression of $v$ nd showed stronger repressor activity than $m s h$ on the Repo expression in most types of glial cells including the LG, whereas $m s h$ showed mild reduction in the Repo expression mainly in the SG, but not in the LG. The repressor activity of vnd started from the initial formation of the LGBs and continued until the glial cells differentiated into mature glia (unpublished data, Fig. 6).

There are several possible explanations for the repressive effect in both loss-of-function 
and gain-of function mutants. First, vnd and EGFR signaling genes together play important roles in establishing identities of the medial and intermediate columns in DV patterning of the ventral neuroectoderm (reviewed by Cornell and von Ohlen, 2000; Skeath and Thor, 2003). Therefore, overexpression of these genes also promote identities of the medial and intermediate in the lateral columns, where many glial cells, including the LG, PG, and some of the SG originate after neurons are formed. This identity change may block glial cell formation and differentiation from the lateral neuroectoderm. Second, overexpression of these genes may also promote neurogenesis over gliogenesis during developmental stages when overexpression was driven by $K r$ - and $s c a-G a l 4$. In addition, repressor activity appears to play a more dominant role than activator activity upon overexpression of $v n d$, as seen in Fig. 4E-H, considering that the DV patterning genes, $v n d$, ind, and $m s h$, act as successive repressors to establish and maintain their identity in the VNE. The results obtained using the loss-of-function and overexpression mutants demonstrate that the expression of a proper level of the DV patterning genes promote identity determination of neurons, while their overexpression represses formation of the glia in the VNE by default. In addition, repressor activity of the DV patterning genes appears to play a dominant role in the establishment of the three columnar divisions along the DV axis.

Similarly, overexpression of the EGFR signaling ligands, Spi and Vn, and the activated form of EGFR signaling receptor, $E g f r^{A C}$, repressed Repo expression in all types of glial cells in the VNE (Figs. 4-6). Thismay be due to the repressor activity of ind, since activation of EGFR signaling induces phosphorylation of ind and $v n d$ to consolidate their repressor activity (Uhler et al., 2007; Moses et al., 2011). In addition, since Egfr overexpression can cause expansion of $v n d$ expression from the medial column to the lateral area (Zhao et al., 2007), the intermediate and lateral columns may have acquired the medial identity, such that the LG and various types of other glia originating from the VNE are not generated after 
overexpression of Spi in the VNE (Fig. 4K, L).

In conclusion, our studies on the glial cell development in the Drosophila VNE revealed that the DV patterning and EGFR signaling genes play prominent roles in promoting neural identity, rather than glial identity during the early stages of CNS development, since their overexpression did not activate glial identity, but rather repressed it. Later, expression of the glial master gene, $\mathrm{gcm}$, is required to promote glial cell identity in the VNE. It appears that the two-step mode of CNS development first ensures generation of a neural circuit and then provides supporting glial cells in the CNS. Our results indicated that the DV patterning genes act locally to promote glial cell formation in their expression domains, but EGFR signaling genes act broadly throughout the VNE. Among the DV patterning genes, vnd, appears to influence glial cell formation and differentiation globally, since it represses ind and $m s h$ to establish and maintain medial identity from the earliest developmental stage. It remains to be investigated how the DV patterning and EGFR signaling genes control the spatial and temporal regulation of glial cell formation and how they interact to promote glial identity in the CNS.

\section{ACKNOWLEDGEMENTS}

The authors are very grateful to Dr. D. M. Mellerick, Dr. A. Nose, Dr. S. Hayashi, and Dr. C.Q. Doe for providing DV patterning gene cDNAs and their mutants. This research was supported by the Basic Science Research Program through the National Research Foundation of Korea (NRF) which was funded by the Ministry of Education, Science and Technology (2009-0074130) and by a grant from the Neurobiology Research Program of the Korean Ministry of Science and Technology, 2004. 
Ashraf SI, Hu X, Roote, J, Ip YT (1999) The mesoderm determinant Snail collaborates with related zinc-finger proteins to control Drosophila neurogenesis. EMBO J 18:6426-6438.

Ashraf SI, Ip YT (2001) The Snail protein family regulates neuroblasts expression of insecutable and string genes involved in asymmetry and cell division in Drosophila. Development 128:4757-4767.

Bhat KM (1999) Segment polarity genes in neuroblast formation and identity specification during Drosophila neurogenesis. BioEssays 21: 472-485.

Barres BA (2008) The mystery and magic of glia: A perspective on their roles in health and disease. Neuron 60:430-440.

Beckervordersandforth, RM, Rickert, C., Altenhein, B., Technau, GM (2008) Subtypes of glial cells in the Drosophila embryonic ventral nerve cord as related to lineage and gene expression. Mech Dev 125:542-557.

Bossing T, Udolph G, Doe CQ, Technau GM (1996) The embryonic nervous system lineages of Drosophila melanogaster. I. Neuroblast lineages derived from the ventral half of the neuroectoderm. Dev Biol 179:41-64.

Brand AH, Perrimon N (1993) Targeted gene expression as a means of altering cell fates and generating dominant phenotypes. Development 118:401-415.

Cai Y, Chia W, Yang X (2001) A family of Snail-related zinc finger proteins regulates two distinct and parallel mechanisms that mediate Drosophila neuroblast asymmetric divisions. EMBO J 20:1704-1714.

Chortard C, Salecker I (2004) Neurons and glia team players in axon guidance. Trends Neurosci 27:655-661.

Chu H, Parras C, White K, Jimenez F (1998) Formation and specification of ventral 
neuroblasts is controlled by vnd in Drosophila neurogenesis. Genes Dev 12:3613-3624.

Cornell RA, von Ohlen T (2000) $v n d / n k x$, ind/gsh, and $m s h / m s x$ : conserved regulators of dorsoventral neural patterning? Curr Opin Neurobiol 10:63-71.

Cowden J, Levine, M (2003) Ventral dominance governs sequential patterns of gene expression across the dorsal-ventral axis of the neuroectoderm in the Drosophila embryo. Dev Biol 262:335-349

D’Alesso M, Frasch M (1996) msh may play a conserved role in dorsoventral patterning of the neuroectoderm and mesoderm. Mech Dev 58:217-231.

Edenfeld G, Stork T, Klämbt C (2005) Neuron-glia interaction in the insect nervous system. Curr Opin Neurobiol 15:34-39.

Freeman MR, Doherty J (2006) Glial cell biology in Drosophila and vertebrates. Trends Neurosci 29:82-90.

Fuse N, Hirose S, Hayashi S (1994) Diploidy of Drosophila imaginal cells is maintained by a transcriptional repressor encoded by escargot. Genes Dev 8:2270-2281.

Golembo M, Raz E, Shilo BZ (1996) The Drosophila embryonic midline is the site of Spitz processing, and induces activation of the EGF receptor in the ventral ectoderm. Development 122:3363-3370.

Halassa MM, Haydon PG (2010) Integrated brain circuits: astrocytic networks modulate neuronal activity and behavior. Annu Rev Physiol 72:335-355.

Hartenstein V (2011) Morphological diversity and development of glia in Drosophila. Glia 59: $1237-1252$.

Hosoya T, Takizawa K, Nitta K, Hotta, Y (1995) glial cells missing: a binary switch between neuronal and glial determination in Drosophila. Cell 82:1025-1036.

Isshiki T, Takeichi M, Nose A (1997) The role of $m s h$ homeobox gene during Drosophila neurogenesis: implication for the dorsoventral specification of the neuroectoderm. 
Development 124:3099-3109.

Ito K, Urban J, Technau GM (1995) Distribution, classification, and development of Drosophila glial cells in the late embryonic and early larval ventral nerve cord. Roux's Arch Dev Biol 204:284-307.

Jimenez F, Martin-Morris LE, Velasco L, Chu H, Sierra J, Rosen DR, White K (1995) vnd, a gene required for early neurogenesis of Drosophila, encodes a homeodomain protein. EMBO J 14:3487-3459.

Jones BW (2001) Glial cell development in the Drosophila embryo. BioEssays 23:877-887.

Jones BW, Fetter RJ, Tear G, Goodman CS (1995) glial cells missing: a genetic switch that controls glial versus neuronal fate. Cell 82:1013-1023.

Klaes A, Menne T, Stollewerk A, Scholz H, Klämbt C (1994) The Ets transcription factors encoded by the Drosophila gene pointed direct glial cell differentiation in the embryonic CNS. Cell 78:149-160.

Kohwi M, Doe CQ (2013) Temporal fate specification and neural progenitor competence during development. Nat Rev Neurosci 14:823-838.

Lehmann R, Tautz D (1994) In situ hybridization to RNA. In: Methods in cell biology, vol. 44 (Goldstein LSB, Fyrberg EA, eds), pp 576-597. San Diego: Academic Press.

Mao H, Lv Z, Ho MS (2012) Gcm proteins function in the developing nervous system. Dev Biol 370:63-70.

McDonald JA, Holbrook S, Isshiki T, Weiss J, Doe CQ, Mellerick DM (1998) Dorsoventral patterning in the Drosophila central nervous system: the vnd homeobox gene specifies ventral column identity. Genes Dev 12:3603-3612.

Mellerick DM, Nirenberg M (1995) Dorsal-ventral patterning genes restrict NK-2 homeobox gene expression to the ventral half of the central nervous system of Drosophila embryos. Dev Biol 171:306-316. 
Miller FD, Gauthier AS (2007) Timing is everything: making neurons versus glia in the developing cortex. Neuron 54:357-369.

Moses C, Helman A, Paroush Z, von Ohlen T (2011) Phosphorylation of Ind by MAP kinase enhances Ind-dependent transcriptional repression. Dev Biol 360:208-215.

O’Neill JW, Bier E (1994) Double in situ hybridization using biotin and digoxigenin tagged RNA probes. Biotechniques 17:873-875.

Parker RJ, Auld, VJ (2006) Roles of glia in the Drosophila nervous system. Sem Cell Dev Biol 17:66-77.

Patel N (1994) Imaging neuronal subsets and other cell types in whole-mount Drosophila embryos and larvae using antibody probes. In: Methods in cell biology. vol. 44 Goldstein LSB, Fyrberg EA, eds), pp 576-597. San Diego: Academic Press.

Rowitch DH, Kriegstein, AR (2010) Developmental genetics of vertebrate glial-cell specification. Nature 468:214-222.

Salter MW, Beggs S (2014) Sublime microglia: expanding roles for the guardians of the CNS. Cell 158:15-24.

Schmid A, Chiba A, Doe CQ (1999) Clonal analysis of Drosophila embryonic neuroblasts: neural cell types, axon projections and muscle targets. Development 126: 4653-4689.

Schmidt H, Rickert C, Bossing T, Vef O, Urban J, Technau, GM (1997) The embryonic central nervous system lineages of Drosophila melanogaster. 2. Neuroblast lineages derived from the dorsal part of the neuroectoderm. Dev Biol 189:186-204.

Schnepp B, Grumbling G, Donaldson T, Simcox A (1996) Vein is a novel component in the Drosophila epidermal growth factor receptor pathway with similarity to neuregulins. Genes Dev 10:2302-2313.

Skeath JB (1999) At the nexus between pattern formation and cell-type specification: the generation of individual neuroblast fates in the Drosophila embryonic central nervous 
system. BioEssays 21:922-931.

Skeath JB, Thor S. 2003. Genetic control of Drosophila nerve cord development. Curr Opin Neurobiol 13:8-15.

Stork T, Bernardos R, Freeman, MR (2012) Analysis of glial cell development and function in Drosophila. In: Cold spring harbor protocols (Zhang B, Freeman, MR, Waddell S, eds), pp 53-74. New York: Cold Spring Harbor Laboratory Press.

Soustelle L, Giangrande A (2007) Glial differentiation and the Gcm pathway. Neuron Glia Biol. 3:5-16.

Uhler J, Zhang H, Syu L-J, Mellerick DM (2007) The Nk-2 box of the Drosophila homeodomain protein, Vnd, contributes to its repression activity in a Grouchodependent manner. Mech Dev 124:1-10.

Verkhratsky A, Parpura V, Rodriguez JJ (2011) Where the thoughts dwell: the physiology of neuronal-glial “diffuse neural net”. Brain Res Rev 66:133-151.

Villegas SN, Poletta FA, Carri NG. (2003) Glia: A reassessment based on novel data on the developing and mature central nervous system. Cell Biol Int 27:599-609.

Vincent S, Vonesch J-L, Giangrande A. 1996. glide directs glial fate commitment and cell fate switch between neurons and glia. Development 122:131-139.

von Hilchen CM, Beckervordersandforth RM, Rickert C, Technau GM Altenhein B (2008) Identity, origin, and migration of peripheral glial cells in the Drosophila embryo. Mech Dev 125:337-352.

Wang W, Chen X, Xu H, Lufkin T (1996) Msx3: a novel murine homologue of the Drosophila msh homeobox gene restricted to the dorsal embryonic central nervous system. Mech Dev 58:203-215.

Weiss JB, Ohlen TV, Mellerick DM, Dressler G, Doe CQ, Scott MP (1998) Dorsoventral patterning in the Drosophila central nervous system: the intermediate neuroblasts 
defective homeobox gene specifies intermediate column identity. Genes Dev 12:35913602.

Zhao G, Wheeler SR, Skeath JS (2007) Genetic control of dorsoventral patterning and neuroblast specification in the Drosophila central nervous system. Int J Dev Biol $51: 107-115$. 


\section{FIGURE LEGENDS}

Figure 1. DV patterning genes are required for the expression of the glial master gene, $\mathrm{gcm}$, from the initial identity determination to differentiation of the LTG. Embryos are doublestained for the glial marker, gcm (blue) and the segmental marker, En (brown). (A) In stage 8 wild type embryos, $\mathrm{gcm}$ expression is detected in the PGBs that are located in each segment (black arrowhead). (C) The number of $\mathrm{gcm}$-positive PGBs is slightly reduced in ind mutant embryos. (B, D, E) The number of $g c m$-positive PGBs is much reduced in $v n d, m s h$, and esg mutant embryos at stage 8. (F) $\mathrm{gcm}$ expression is detected both in the PGBs (arrows) and in the LGBs (empty arrows) in stage 10 wild type embryos. (G, I) The number of gcm-positive LGBs (empty arrowheads) is much reduced in $v n d$ and $m s h$ embryos at stage 10 . (H) The number of $\mathrm{gcm}$-positive LGBs is almost intact in ind embryos. (J) Interestingly, $\mathrm{gcm}$ expression is reduced mainly in PGBs of esg embryos. (K) In stage 13 wild type embryos, gcm expression is detected in the divided LG (white arrowhead) and in the laterally stretched SG (white arrows) in each segment. (L, N) Most of the bilaterally symmetric gcm-positive LG and SG in each segment are reduced in vnd and msh embryos. (M) ind embryos show almost intact $\mathrm{gcm}$ expression in the LG and SG. (O) The number of $\mathrm{gcm}$-positive SG (white arrowheads) is mainly reduced in esg embryos. In all panels, anterior is to the left, and ventral views are shown with the ventral midline marked by black lines. Each segment is marked with anti-En antibody staining (brown).

Figure 2. DV patterning genes are locally required for Repo expression which is essential for differentiation of the LTG in the VNE. (A) In wild type embryos, Repo expression starts in the LGBs (black arrowheads) of each segment at stage 11, and then continues in the divided LG and the laterally stretched NG (arrow) at stage 12 (F). (K) Repo is expressed in the LG (white arrows) located along the longitudinal tracts, the NG and SG in the entire VNC, and 
the PG (black arrows) stretched out dorsally, as shown in each segment at stage 14. (B, D) The number of Repo-expressing LGBs is much reduced in $v n d$ and $m s h$ embryos (asterisk) at stage 11. (E) In esg embryos, the number of Repo-expressing LGBs (asterisk) is slightly reduced. (C) In contrast, the number of Repo-expressing LGBs is not changed in the ind embryos. (G) The numbers of divided Repo-expressing LG and NG are much reduced in the vnd embryos at stage 12. (L) The numbers of Repo-expressing LG along the longitudinal tracts (white arrows) and SG are reduced, such that the VNC becomes narrower in the vnd embryos than in the wild type embryos at stage 14. In addition, the number of Repo-positive PG in some segments of the lateral ectoderm (arrows) is reduced. $(\mathrm{H})$ In stage 12 ind embryos, the numbers of Repo-positive LG and SG are not much reduced compared to wild type embryos. (M) However, many Repo-positive SG are absent in the ind embryos, but the LG along the longitudinal tracts and the PG in the segmental nerve remain intact at stage 14 . (I) The LG do not form properly, but additional SG are formed in the lateral ectoderm in stage 12 msh embryos. (N) In stage 14 msh embryos, some of the Repo-positive LG are missing in the longitudinal tracts (white arrow). (J) In stage 12 esg embryos, the number of Repo-positive SG is reduced, particularly in the lateral side (arrow). (O) In stage 14 esg embryos, the number of Repo-positive PG is markedly reduced in the lateral neuroectoderm (arrows). In all panels, anterior is to the left, and ventral views are shown with the ventral midline marked by black and white lines. Each segment is marked with anti-En antibody staining (brown).

Figure 3. EGFR signaling genes are broadly required for identity determination and differentiation of the LTG in the VNE. (A-D) $\mathrm{gcm}$ expression at stage 10. (A) $\mathrm{gcm}$ expression is detected in the LGBs (black pointed arrowhead) and PGBs (white pointed arrowhead) in stage 10 wild type embryos. (B) gcm-positive LGBs and PGBs are not affected in stage 10 
sim embryos. (C, D) The numbers of $g c m$-positive LGBs and PGBs are much reduced in Egfr and spi mutant embryos. Repo expression at stage 11 (E-H), stage 12 (I-L) and stage 16 (MP). (E, I, M) In wild type embryos, Repo expression starts in the LGBs (black arrowhead) at stage $11(\mathrm{E})$, detected in many LG and SG that are stretched out in the lateral region (white brackets) at stage 12 (I) and in all types of glia including the PG at stage $16(\mathrm{M})$. (F, J, N) In sim embryos, Repo-positive LGBs remain intact at stage 11 (F). Later at stage 12, the number of SG is reduced (J) and the longitudinal tracts where the LG is located are collapsed at the midline (white arrowhead) at stage $16(\mathrm{~N}) .(\mathrm{G}, \mathrm{K}, \mathrm{O})$ In Egfr embryos, the number of Repopositive LGBs is much reduced at stage 11, and the number of all types of Repo-positive glia is virtually absent in some segments (white and black arrows) at stages 12 and $16(\mathrm{~K}, \mathrm{O}) .(\mathrm{H}$, L, P). In spi embryos, the number of Repo-positive LGBs is reduced in some of the segments $(\mathrm{H})$, and some of the Repo-expressing NG are missing (white brackets) at stage 12 (L). At stage 16, the numbers of Repo-expressing SG and PG are markedly reduced except for the LG along the longitudinal tracts (white arrows). In all panels, anterior is to the left, and ventral views are shown with the ventral midline marked by black lines. Each segment is marked with anti-En antibody staining (brown).

Figure 4. Overexpression of the DV patterning and EGFR signaling genes represses the formation of Repo-expressing LTG. (A) In wild-type embryos, Repo expression (green) is detected in the LG, SG, and PG at stage 15. (B) Activated MAPK (red) is expressed in the longitudinal tracts, midline cells, segmental nerves, and junctions between muscle and dorsal epidermis (white arrow). (C) Merged ventral view of Repo and activated MAPK. (D) Merged side view of expressions of Repo and activated MAPK. (E) In stage $15 \mathrm{Kr}$-Gal4/UAS-vnd embryos, the number of all types of Repo-positive glia is severely reduced in the $K r$ expressing central region of the embryos (white arrow). (F) Expression of activated MAPK is 
also severely reduced in the central region as well as other regions of the embryos. $(\mathrm{G})$ Merged ventral view of expressions of Repo and activated MAPK. (H) Merged side view of expressions of Repo and activated MAPK. (I) In stage $15 \mathrm{Kr}$-Gal4/UAS-spi embryos, the number of Repo-positive SG is much reduced, although some of the Repo-positive LG and PG remain. (J) Expression of activated MAPK is also severely reduced in the entire VNE of the embryos. (K) Merged ventral view of expressions of Repo and activated MAPK. (L) Merged side view of expressions of Repo and activated MAPK. The number of Repo-positive SG is much reduced in the ventral side of the ventral nerve cord. In all panels, anterior is to the left. In all panels except for D, H, and L, ventral views are shown with the ventral midline marked by white lines. In panels $\mathrm{D}, \mathrm{H}$, and L, side views are shown.

Figure 5. Repression of Repo expression in the LGBs by overexpression of the DV patterning gene, $v n d$, is due to the identity change in the intermediate and lateral columns. (A, G) In stage 11 wild type embryos, vnd RNA (green) is expressed in the medial neurons (white arrow) in the VNE. (B, H) Repo expression (red) starts to be detected in the LGBs. (C, I) Merged views of the expression of $v n d$ RNA (green) and Repo (red). (D, J) In stage $11 \mathrm{Kr}$ Gal4/UAS-vnd embryos, vnd RNA is expressed in the mesoderm region of the embryos besides the medial neurons (white arrows). (E, K) Repo-positive LGBs are reduced in the $\mathrm{Kr}$ expressing middle region of the VNE (two white arrowheads). (F, L) Merged views of the expression of vnd RNA (green) and Repo (red). In all panels, anterior is to the left. In panels A-F, ventral views are shown with the ventral midline marked by white lines. In panels G-L, side views are shown.

Figure 6. The defects in the determination of the DV identity cause repression of Repo expression in the LTG. (A-D) In wild type stage 15 embryos, Repo (green) is expressed in the 
LG, SG, and PG, and activated MAPK (red) is expressed in the segmental boundaries (white arrowheads). (E-L) In stage $15 K r$-Gal4/UAS-Egfr ${ }^{A C}$ and $K r$-Gal4/UAS-spi embryos, expressions of Repo and activated MAPK are severely repressed in the $K r$-expressing middle region of the embryos (white arrows). (M-T) In stage $15 \mathrm{Kr}$-Gal4/UAS-vnd and $\mathrm{Kr}$ Gal4/UAS-msh embryos, expressions of Repo and activated MAPK are also repressed in the $K r$-expressing middle region of the embryos (white arrows). In all panels, anterior is to the left. In panels D, H, L, P, T, side views are shown. In panels A-C, E-G, I-K, M-O, Q-S, ventral views are shown with the ventral midline marked by white lines. 
Dorsoventral patterning genes

\section{EGFR}
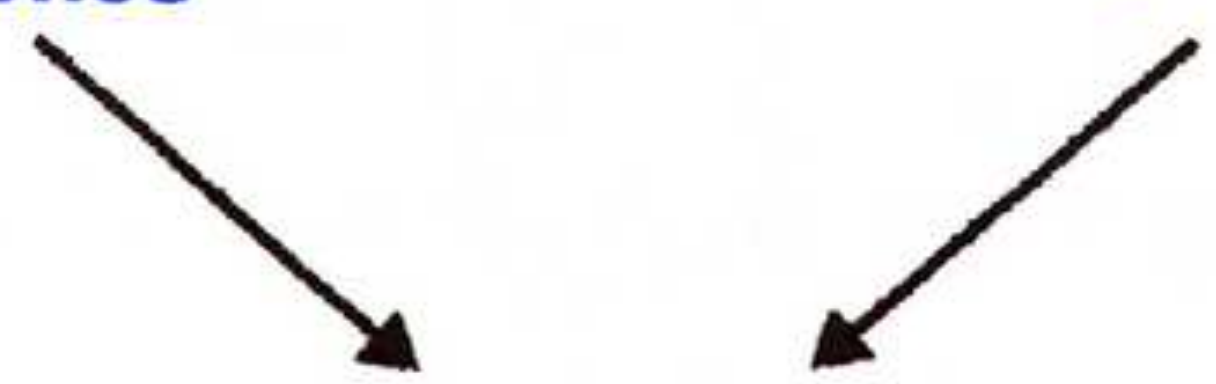

$\mathrm{gcm}$
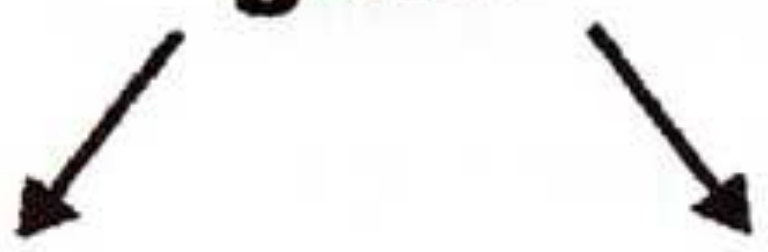

ttk, factor $X$

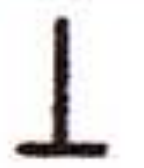

Neuronal fate

$\leftarrow$

Glial fate

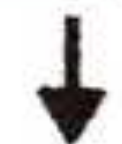

1

repo, pnt

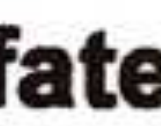


Click here to download high resolution image
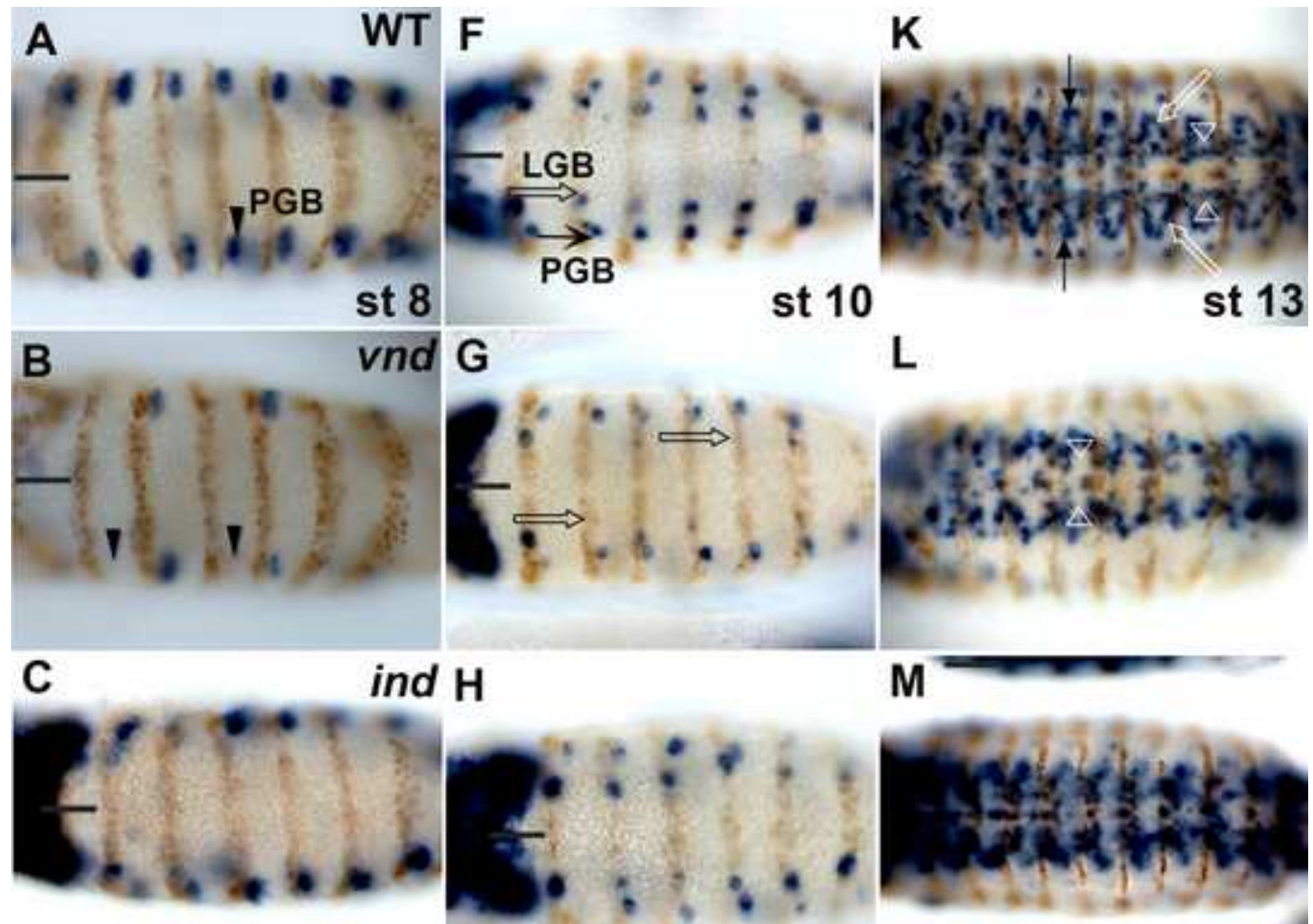

ind $\mathrm{H}$

M
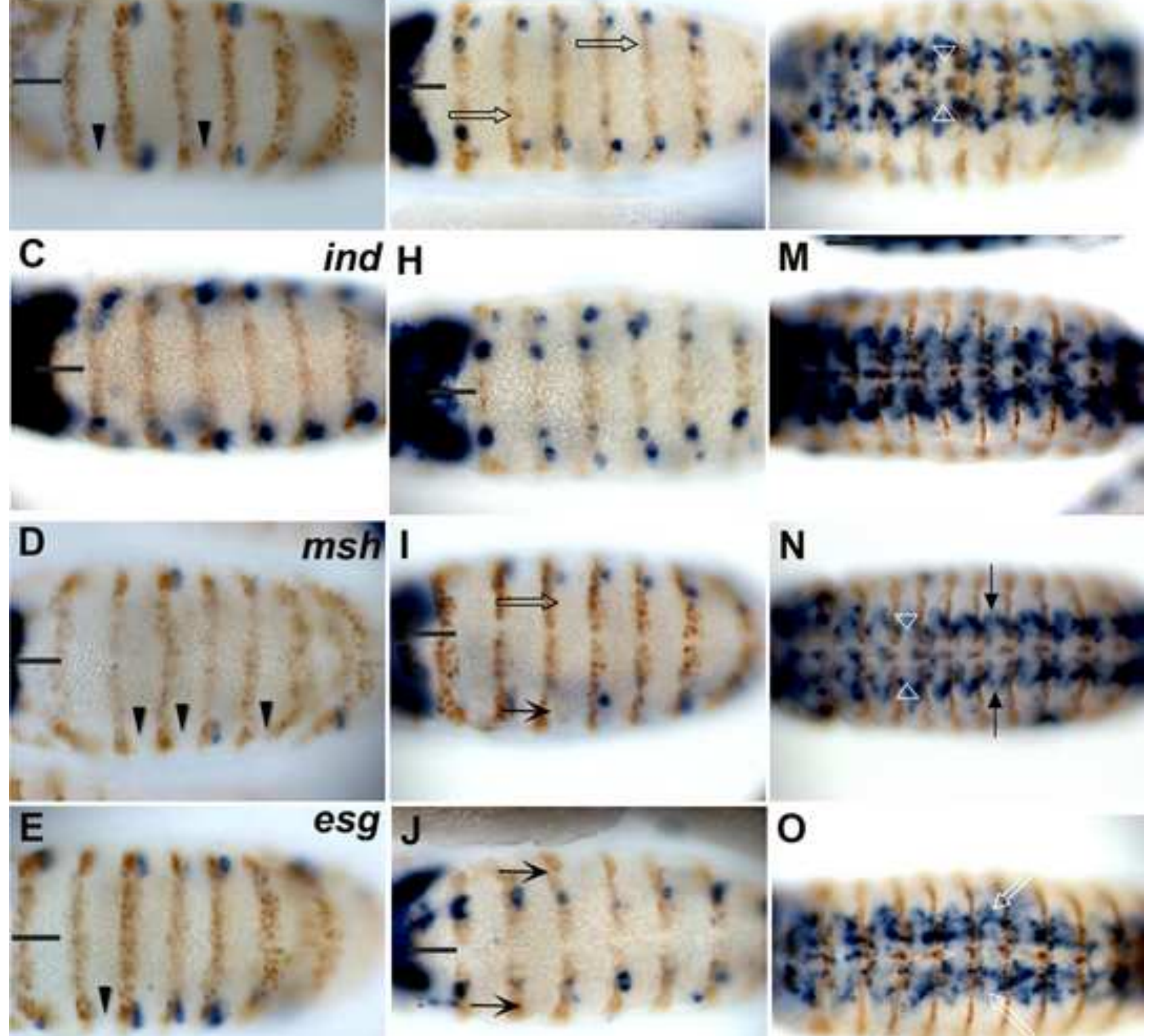

N
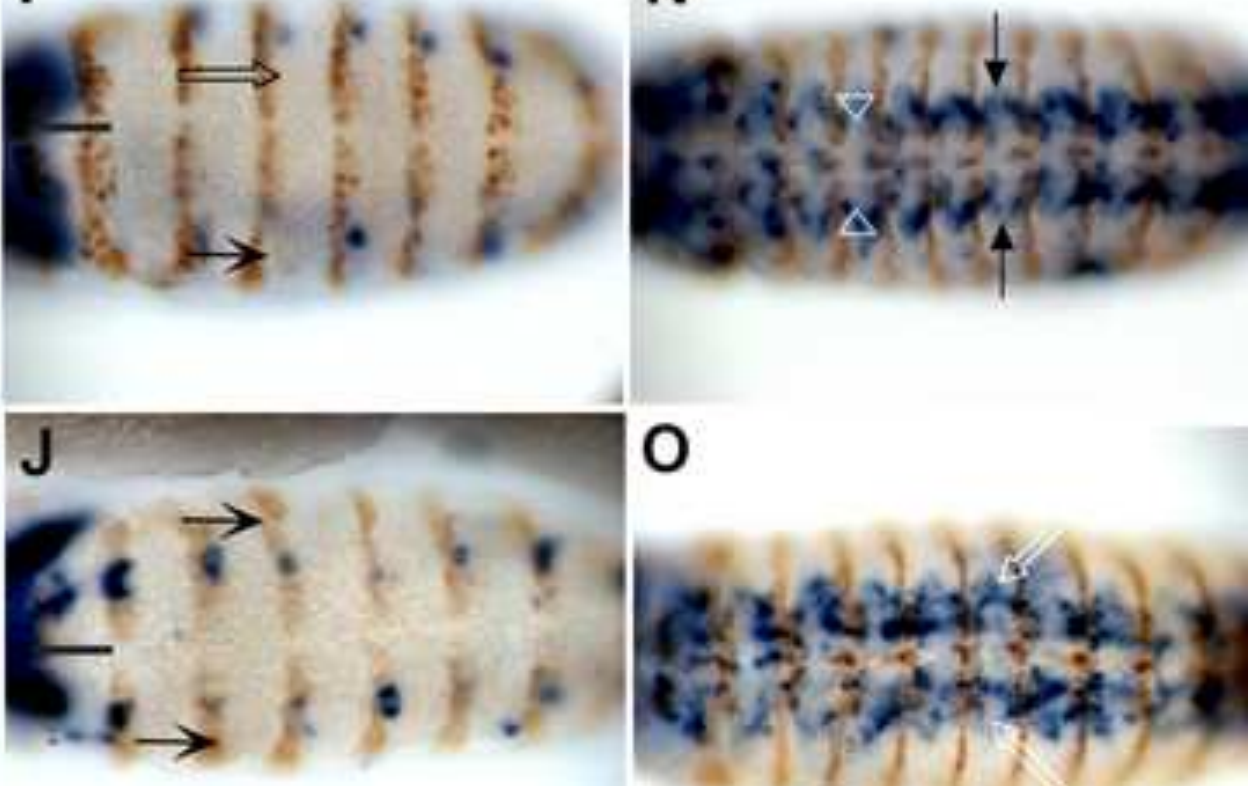

0

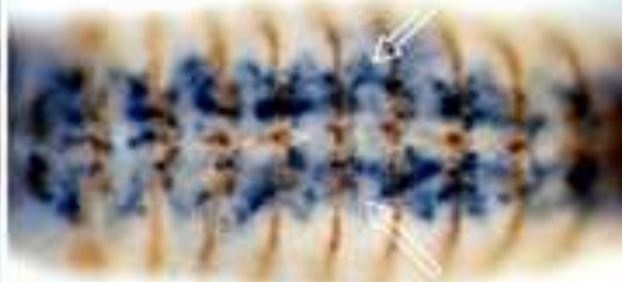




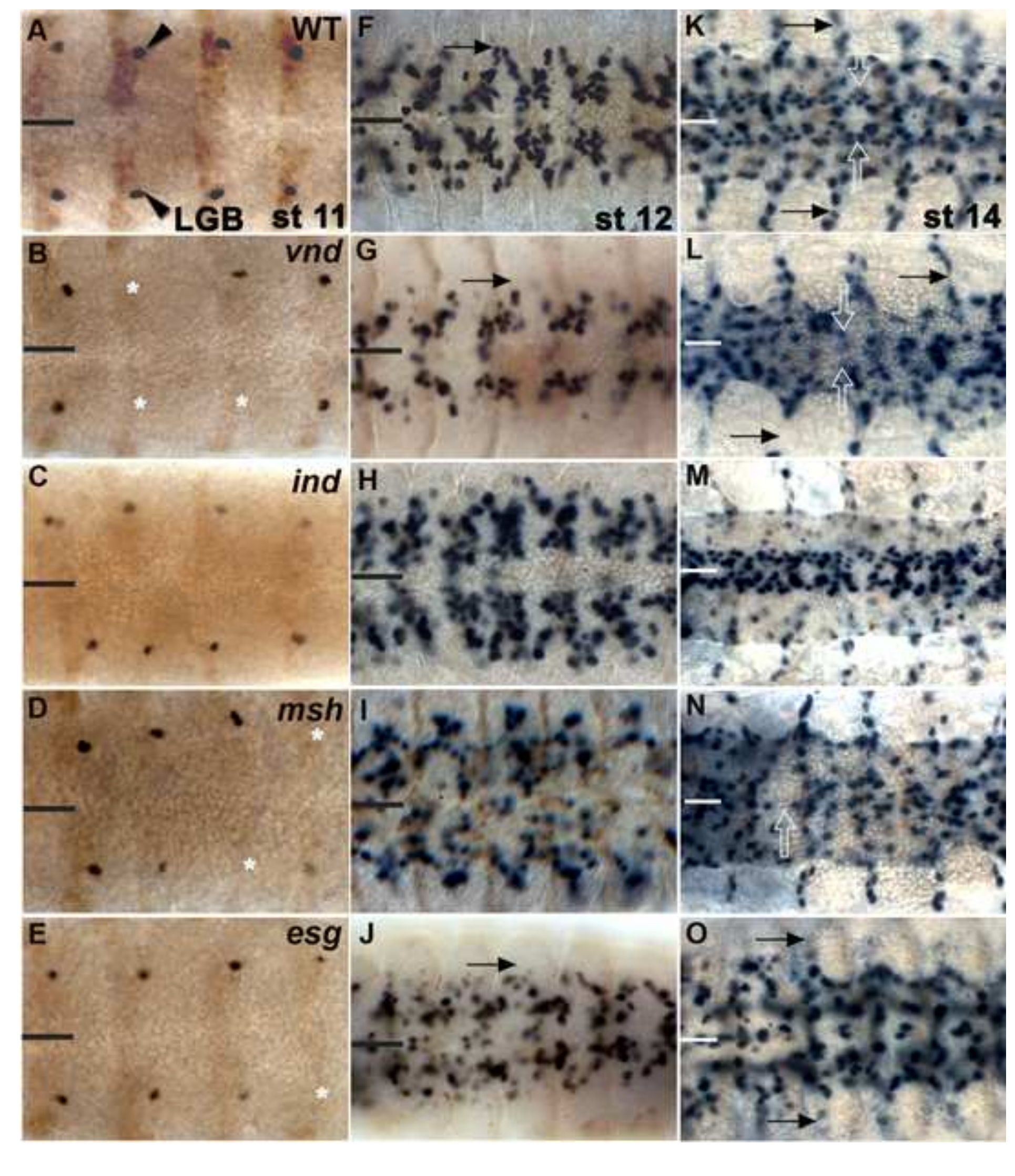

Click here to download high resolution image

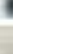

.


Click here to download high resolution image
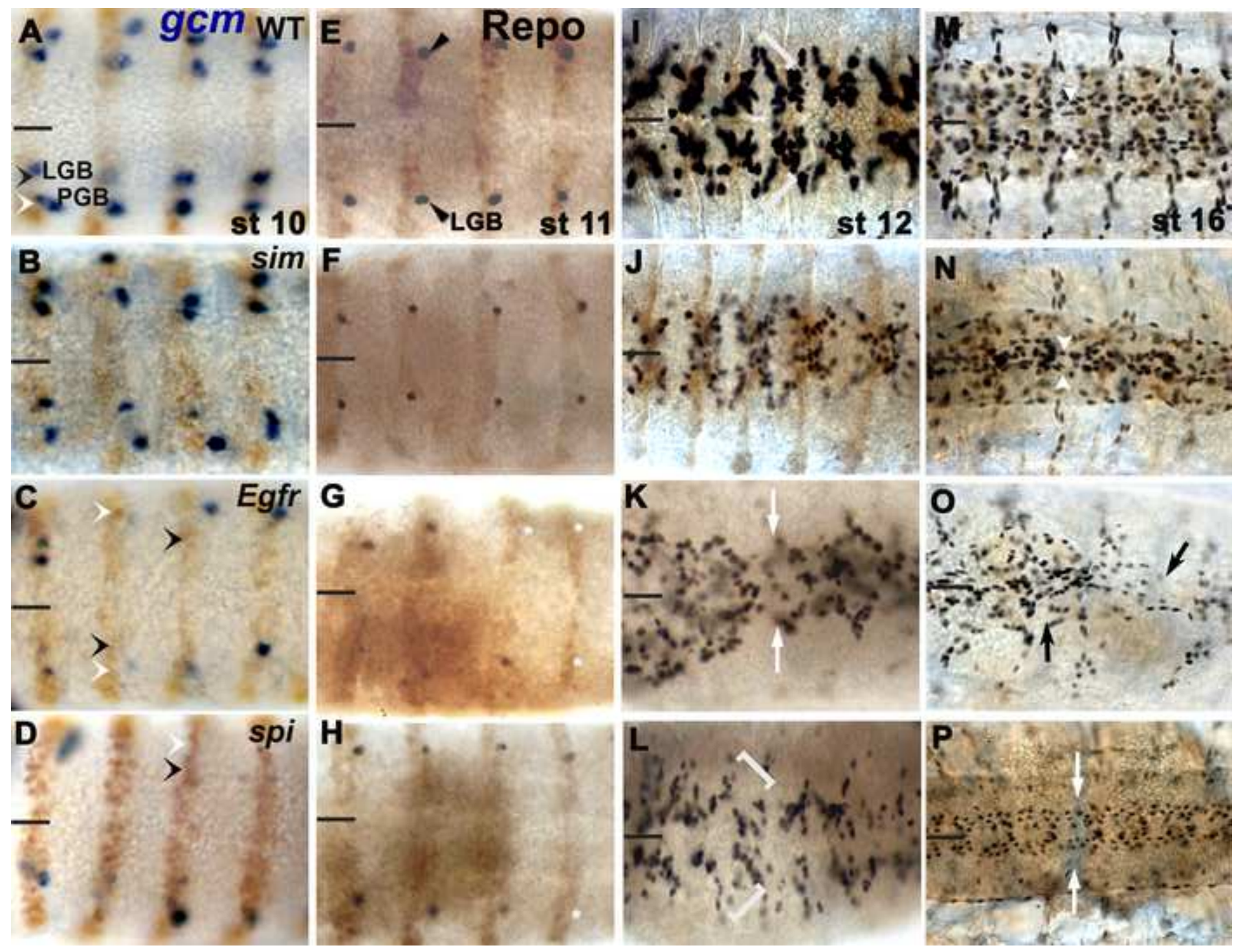


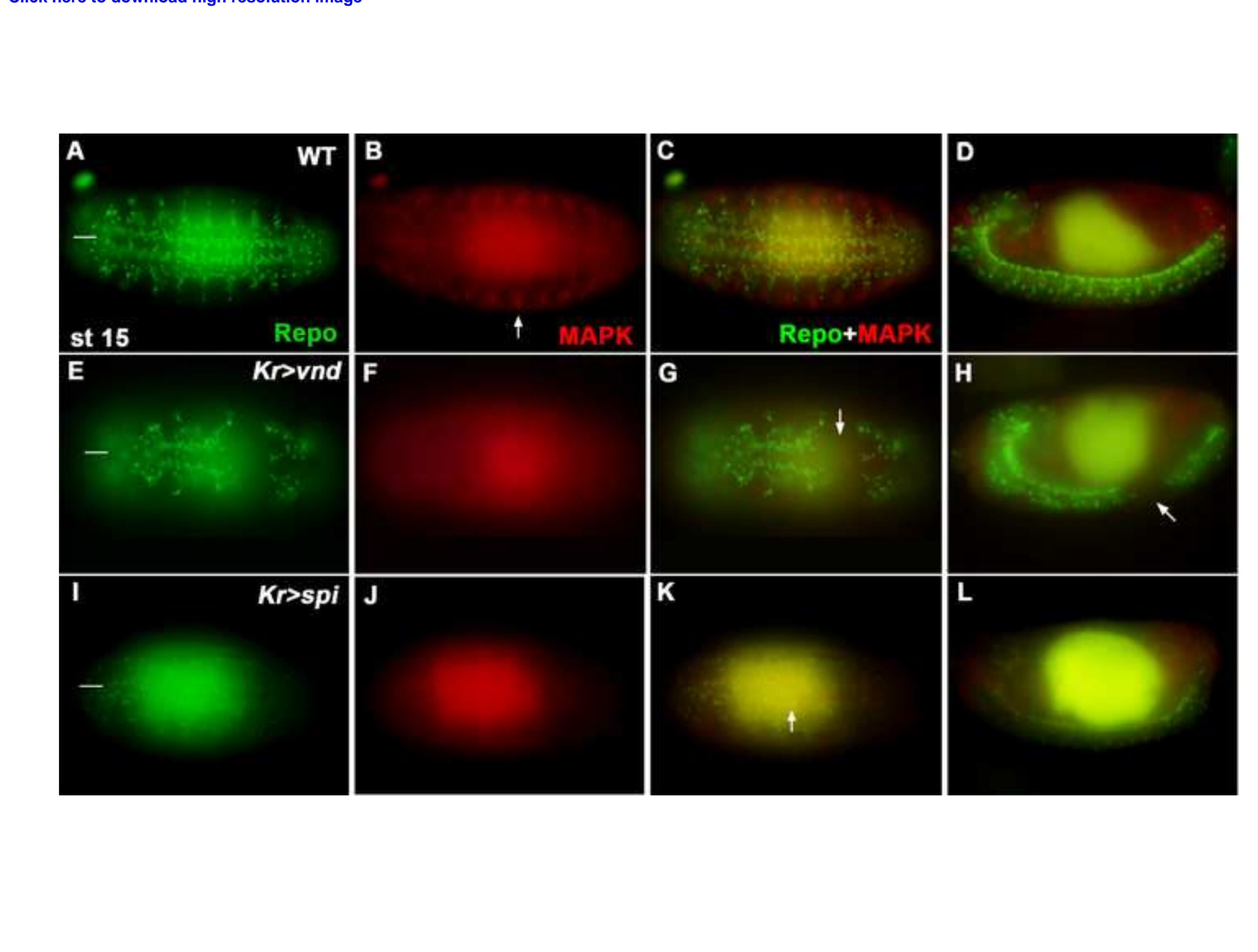

Click here to download high resolution image 


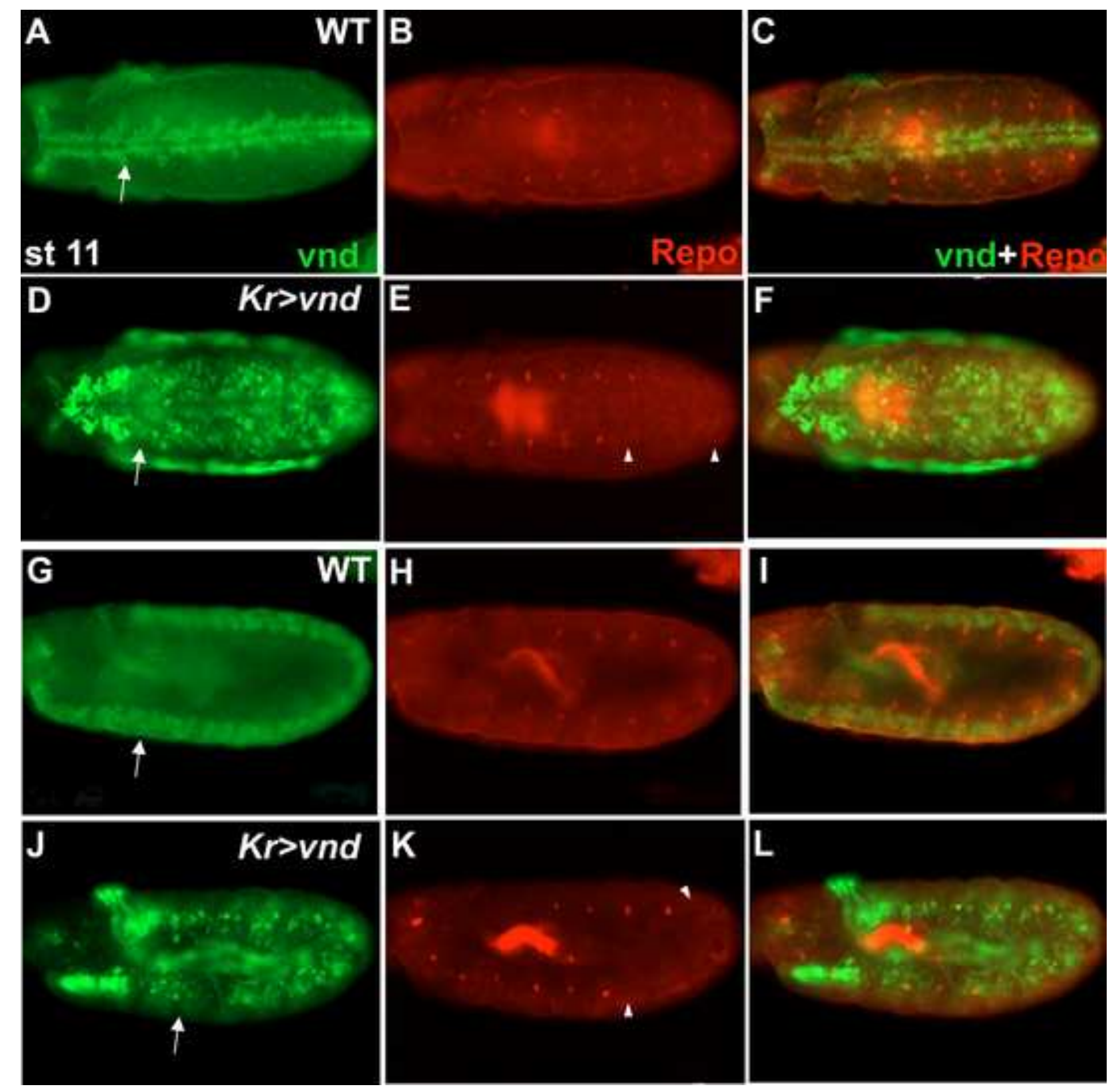

Figure 5
Click here to download high resolution image 
Click here to download high resolution image

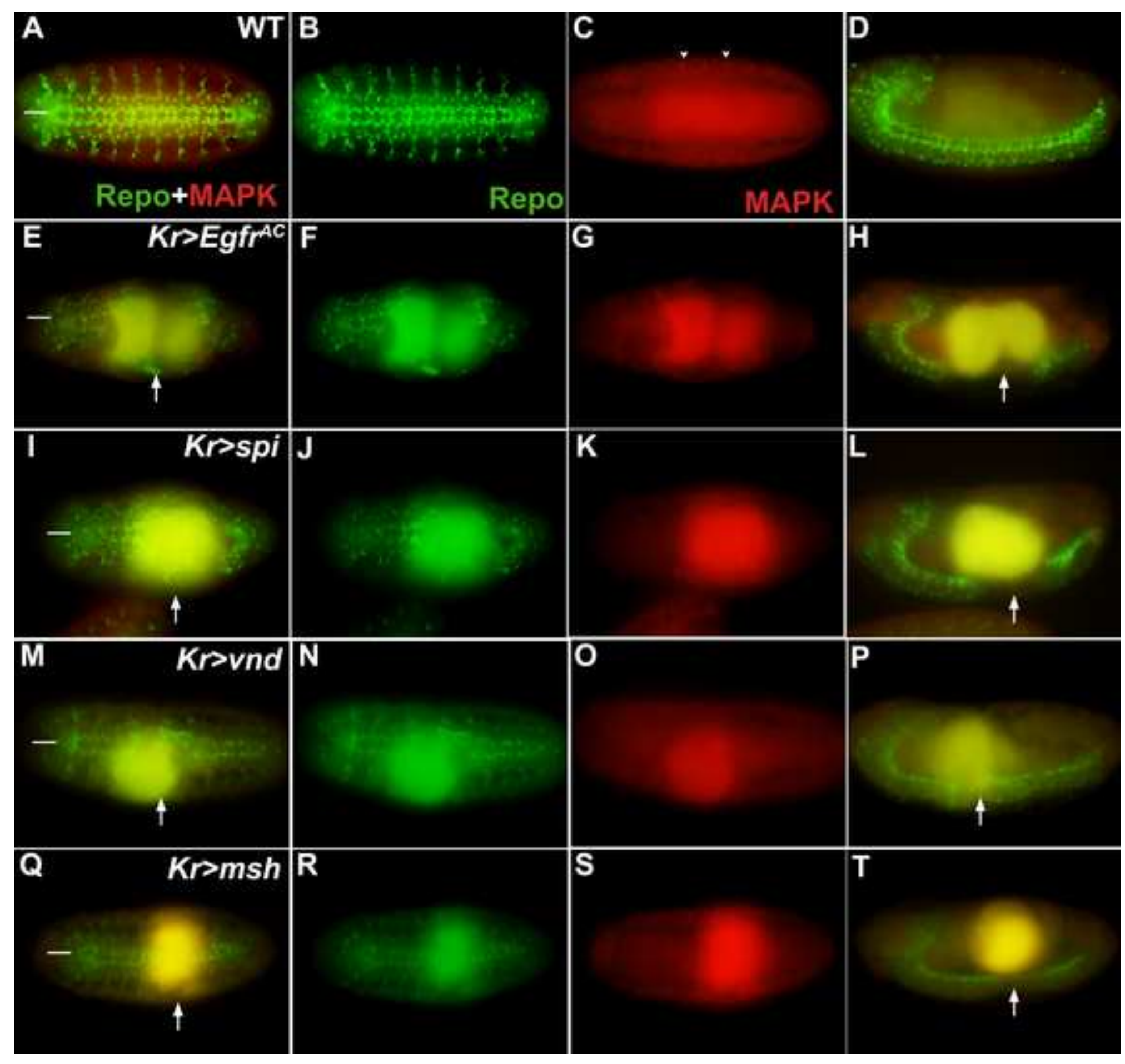

